\title{
CARBON MONOXIDE IN COLLAPSING INTERSTELLAR CLOUDS
}

\author{
T. De Jong, Shih-I Chu,* and A. Dalgarno \\ Center for Astrophysics, Harvard College Observatory and Smithsonian Astrophysical Observatory \\ Received 1974 August 12; revised 1974 December 30
}

\begin{abstract}
We have calculated the energy loss rates, the brightness temperatures and the profiles of lines of carbon monoxide in collapsing interstellar clouds. The most recent data for the $\mathrm{H}_{2}-\mathrm{CO}$ collision rates have been used in the calculations; a useful extrapolation of these data to high $j$ levels is given. The density distribution and the velocity field inside the cloud are varied, one model applying to a cloud of uniform density that is collapsing in free fall, and a second to a cloud in isothermal collapse. Analytical relations for total $\mathrm{CO}$ cooling at low and high densities are derived. The effects of varying the cross sections and the density distribution, the velocity field, and the geometry of the clouds are discussed. The line profiles are a sensitive tool for testing the models against the observations. The observed CO lines can be explained by collapsing models in which both density and velocity gradients occur. The intensity ratio of the $j=2 \rightarrow 1$ and the $j=1 \rightarrow 0 \mathrm{CO}$ lines in Orion is consistent with such models. We predict that $\mathrm{CO}$ infrared lines from dense interstellar clouds should be observable with instruments of high spectral resolution at wavelengths longward of 200 microns.
\end{abstract}

Subject headings: molecules, interstellar — nebulae

\section{INTRODUCTION}

By extending the methods developed by Sobolev (1960), Castor (1970), and Lucy (1971), Scoville and Solomon (1974) and Goldreich and Kwan (1974) have solved the problem of radiative transfer in a collapsing gas cloud of uniform density in the limit when the thermal velocity is small compared with the collapse velocity. They have demonstrated that the coupling of radiative trapping and collisional excitation and deexcitation is critical to the interpretation of the observed intensities of molecular rotational lines in dense interstellar clouds. In order to study the sensitivity of the results to the density and velocity distributions, we have constructed an alternative model of an interstellar cloud in which the density varies as the inverse square of the distance and the collapse velocity is constant. In this paper we compare its consequences with those of the previous models. We also test the sensitivity of all the models to the collisionalexcitation rate coefficients.

\section{MULTILEVEL LINE TRANSFER}

We consider the transfer of line radiation of multilevel molecules in a spherical cloud. Let the radial coordinate be $r$ and suppose $n_{i}(r) \mathrm{cm}^{-3}$ is the population of level $i$ at the point $r$. In statistical equilibrium,

$$
n_{i}(r) \sum_{j} P_{i j}(r)=\sum_{j} n_{j}(r) P_{j i}(r),
$$

where $P_{i j}(r)$ is expressible in terms of the Einstein radiative transition probabilities $A_{i j}$ and $B_{i j}$ and the

* Also Department of Chemistry, Harvard University, Cambridge, Massachusetts 02138. collision-induced transition probabilities $C_{i j}$ in the form

$$
\begin{aligned}
P_{i j}(r) & =A_{i j}+B_{i j}\left\langle J_{i j}(r)\right\rangle+C_{i j}(r) & & (i>j) \\
& =B_{i j}\left\langle J_{i j}(r)\right\rangle+C_{i j}(r) & & (i<j),
\end{aligned}
$$

$\left\langle J_{i j}(r)\right\rangle$ being the mean integrated radiation field at the frequency $\nu_{i j}$ corresponding to the transition from level $i$ to level $j$. The mean radiation field can be obtained from the equations of transfer in integral form

$$
\left\langle J_{i j}(\boldsymbol{r})\right\rangle=\int \mathrm{K}_{i j}\left(\left|\boldsymbol{r}-\boldsymbol{r}^{\prime}\right|\right) S_{i j}\left(\boldsymbol{r}^{\prime}\right) d \boldsymbol{r}^{\prime},
$$

where $\mathrm{K}_{i j}$ is a kernel embodying the physics of the situation, $S_{i j}(\boldsymbol{r})$ is the line source function (cf. Castor 1970), and the integration is carried out over a spherical cloud. Equation (3) is to be solved subject to the boundary condition that outside the cloud $\left\langle J_{i j}(r)\right\rangle$ equals the Planck function $B_{i j}\left(T_{\mathrm{BB}}\right)$ evaluated at the temperature $T_{\mathrm{BB}}=2.7 \mathrm{~K}$ of the universal blackbody radiation field.

The transfer equation simplifies considerably in the presence of large velocity gradients when the velocities are large compared with the thermal velocities $v_{t}$ (Sobolev 1960; Castor 1970; Lucy 1971). Thus, in a spherically symmetric cloud of radius $R$ with a large velocity gradient, photons emitted at some point $P$ in the cloud are absorbed only within a small distance from $P$ of the order $l \approx v_{t} R / V \ll R$ where $V$ is a typical large-scale velocity and $v_{t}$ is the thermal velocity of the molecules. Because a photon cannot be absorbed by molecules that are more than a Doppler width away in frequency, any photon that travels beyond the distance $l$ will escape, provided that in addition the 
velocity $v$ is a monotonic function of $r$. With the further assumption of complete redistribution of frequencies, the source function can be taken out of the integral, and the transfer equation may be written

$$
\left\langle J_{i j}(r)\right\rangle=\left[1-\beta_{i j}(r)\right] S_{i j}(r)+\beta_{i j}(r) B_{i j}\left(\nu_{i j}, T_{\mathrm{BB}}\right),
$$

where $\beta_{i j}(r)$ is the probability that a photon emitted in the transition from level $i$ to level $j$ at the radial distance $r$ will escape. If all the photons escape, $\beta_{i j}=1$ and $\left\langle J_{i j}(r)\right\rangle$ is the blackbody radiation field; if none escape $\left\langle J_{i j}(r)\right\rangle$ is the local source function $S_{i j}(r)$.

The source function $S_{i j}(r)$ can be written

$$
S_{i j}(r)=\frac{2 h \nu_{i j}^{3}}{c^{2}}\left[\frac{n_{j}(r) g_{i}}{n_{i}(r) g_{j}}-1\right]^{-1}
$$

where $g_{i}$ and $g_{j}$ are the statistical weights of levels $i$ and $j$, respectively.

To obtain an expression for the escape probability $\beta_{i j}(r)$, we adopt an argument due to Rybicki (1970). We introduce the optical depth $t(\nu, r, \mu)$ at the point $P$ at frequency $\nu$ obtained by integrating from $P$ to the boundary of the sphere along a ray $s$ that makes an angle $\cos ^{-1} \mu$ with the radius vector to the point $P$. Then

$$
t(\nu, r, \mu)=\int k_{0}(r, s, \mu) \phi\left[\nu-\nu_{0}+\frac{\nu_{0} s}{c} \frac{d v_{s}}{d s}(r, \mu)\right] d s,
$$

where the integrand is the absorption coefficient, written as a product of the absorption coefficient at the center of the line $\nu=\nu_{0}$ and the normalized line profile $\phi(\nu)$ and it is assumed that the velocity gradient $d v_{s} / d s$ is constant over the range of $s$ that contributes significantly to the integral. The absorption coefficient for the $i-j$ transition is given by

$$
k_{0}=\frac{h \nu_{0}}{4 \pi}\left(n_{j} B_{j i}-n_{i} B_{i j}\right) .
$$

If the velocity gradient is large, $\phi$ is strongly peaked at $s=0$ and

$$
t(\nu, r, \mu)=k_{0}(r) \int_{0}^{\infty} \phi\left(\nu-\nu_{0}+\frac{\nu_{0}}{c} \frac{s d v_{s}}{d s}\right) d s .
$$

Making the substitutions, $x=\nu_{0}-\nu$ and $x^{\prime}=$ $-x+\alpha_{0} \nu_{0} s / c$, where $\alpha_{0}(r, \mu)=d v_{s} / d s$ evaluated at $s=0$, we obtain

$$
t(x, r, \mu)=k_{0}(r) \frac{c}{\alpha_{0} \nu_{0}} \int_{-x}^{+\infty} \phi\left(x^{\prime}\right) d x^{\prime} .
$$

The escape probability in the line, averaged over all angles, is

$$
\beta(r)=\int_{4 \pi} \frac{d \Omega}{4 \pi} \int_{-\infty}^{\infty} d x \phi(x) \exp [-t(x, r, \mu)] .
$$

Changing the variable to

$$
y=\int_{-\infty}^{x} \phi\left(x^{\prime}\right) d x^{\prime}
$$

and using the normalization condition $\int_{-\infty}^{\infty} \phi(x) d x=1$, we obtain

$$
\beta(r)=\frac{1}{2} \int_{-1}^{+1} d \mu\left\{\frac{1-\exp [-\tau(r, \mu)]}{\tau(r, \mu)}\right\},
$$

where $\tau(r, \mu)=k_{0}(r) c / \nu_{0} \alpha_{0}(r, \mu)$.

In spherical geometry,

$$
\frac{d v_{s}(r, \mu)}{d s}=\mu^{2} \frac{d v(r)}{d r}+\left(1-\mu^{2}\right) \frac{v(r)}{r} ;
$$

and we recover the expression of Castor (1970) for the escape probability.

\section{CLOUD MODELS}

We have carried out calculations for two cloud models which for purposes of comparison we take to have the same mass $M$, the same radius $R$ (and therefore the same average density $n$ ), and the same velocity $V$ at the outer boundary. In the first cloud model, the cloud is contracting spherically with a constant radial velocity and the density is varying inversely as the square of the distance. The model is an asymptotic solution of the isothermal collapse of a spherical cloud (Larson 1969). Although there is no velocity gradient in the radial direction, the spherical contraction causes a velocity gradient in all other directions and $d v_{s} / d s$ is a function of $r$ and $\mu$. The local solution is limited to angles such that $1-\mu^{2} \geq$ $v_{t} / V$. As a typical example consider molecules of carbon monoxide at a temperature of $80 \mathrm{~K}$. If $V \sim 5 \mathrm{~km} \mathrm{~s}^{-1}$, $\mu^{2} \leq 0.95$ and the angle must be greater than $10^{\circ}$. Photons are emitted isotropically so that the adopted solution underestimates the escape probability in perhaps 1 percent of the escape events. The analysis is also strictly correct only to first order in the velocity gradient.

Our second cloud model is that of Goldreich and Kwan (1974). It describes a spherical cloud of uniform density collapsing in free-fall. Scoville and Solomon (1974) have explored a similar model of a planeparallel cloud with uniform density. In plane-parallel geometry $d v_{s} / d s=\mu^{2}(d v / d z)$, where $z$ is the depth normal to the plane. Applying the Eddington approximation which is equivalent to replacing $\mu^{2}$ by the average of $\frac{1}{3}$, Scoville and Solomon obtain for the escape probability

$$
\beta_{i j}=\frac{1-\exp \left(-3 \tau_{i j}\right)}{3 \tau_{i j}},
$$

and the numerical results at large values of $\tau_{i j}$ differ from those of the spherical models.

In summary, the parameters of the two spherical models are: for model $\mathrm{A}$ of Goldreich and Kwan 
(1974), the $\mathrm{H}_{2}$ density $n$ is constant, the velocity is given by $v(r)=r V \mid R, \tau_{i j}$ is given by

$$
\tau_{i j}=\frac{A_{i j} c^{3} n_{i}}{8 \pi v_{i j}{ }^{3}}\left(\frac{n_{j} g_{i}}{n_{i} g_{j}}-1\right) \frac{R}{V},
$$

and $\beta_{i j}$ by $\beta_{i j}=\left[1-\exp \left(-\tau_{i j}\right)\right] / \tau_{i j}$; in model B,

$$
\begin{gathered}
n(r)=\frac{1}{3} n(r / R)^{-2}, \quad v(r)=V, \\
\tau_{i j}(r, \mu)=\frac{A_{i j} c^{3} n_{i}}{8 \pi v_{i j}{ }^{3}}\left(\frac{n_{j} g_{i}}{n_{i} g_{j}}-1\right) \frac{r}{V\left(1-\mu^{2}\right)},
\end{gathered}
$$

and

$$
\beta_{i j}(r)=\int_{0}^{1} d \mu \frac{1-\exp \left[-\tau_{i j}(r, \mu)\right]}{\tau_{i j}(r, \mu)}
$$

\section{COLLISION CROSS SECTIONS}

Various assumptions have been made about the collision cross sections that determine the coefficients $C_{i j}$ in equation (2). In the calculation of the populations of the rotational levels $j$ of carbon monoxide, the required cross sections $\sigma\left(j-j^{\prime}\right)$ are those for the collisions

$$
\mathrm{H}_{2}+\mathrm{CO}(j) \rightarrow \mathrm{H}_{2}+\mathrm{CO}\left(j^{\prime}\right) .
$$

Goldsmith (1972) considered a variety of cross section estimates which varied slowly with $j$ or $j^{\prime}$, were independent of energy, and were of the order of $10^{-15}$ $\mathrm{cm}^{2}$. Similar assumptions but with a cross section of $5 \times 10^{-17} \mathrm{~cm}^{2}$ were made by Glassgold and Langer (1973). Scoville and Solomon (1974) adopted the cross sections derived by Compaan et al. (1973) from measured widths of rotational Raman lines, which vary from $2.7 \times 10^{-16} \mathrm{~cm}^{2}$ for the $0-1$ transition to $6.6 \times 10^{-17} \mathrm{~cm}^{2}$ for the $0-5$ transition and are nearly

\begin{tabular}{|c|c|c|}
\hline$j-j^{\prime}$ & $a\left(\mathrm{~cm}^{3} \mathrm{~s}^{-1}\right) \times 10^{10}$ & $b$ \\
\hline $\begin{array}{l}1 \ldots \ldots \cdots \cdots \cdots \\
2 \ldots \ldots \cdots \cdots \cdots \\
4 \ldots \ldots \cdots \cdots \\
5 \ldots \ldots \ldots m b\end{array}$ & $\begin{array}{l}1.66 \\
2.80 \\
1.19 \\
1.00 \\
1.30\end{array}$ & $\begin{array}{l}1.67 \\
1.47 \\
1.85 \\
1.55 \\
2.24\end{array}$ \\
\hline
\end{tabular}
independent of the initial rotational quantum number,
TABLE 1

Constants in the Representation (17) of the Collision Rate Coefficients $K\left(j-j^{\prime}\right)$

whereas Goldreich and Kwan (1974) assumed that for collisions in which

$$
j^{\prime}<j, \sigma\left(j-j^{\prime}\right)=2.5 \times 10^{-15}(h B / k T) \mathrm{cm}^{2},
$$

where $B$ is the rotational constant of $\mathrm{CO}$. Gerola and Sofia (1975) used cross sections $\sigma\left(j-j^{\prime}\right)=10^{-15} \mathrm{~cm}^{2}$ and $\sigma\left(j-j^{\prime}\right)=0$ depending upon the values of $\left(j-j^{\prime}\right)$.

We have based our selection of cross sections on the close-coupling calculations of Green and Thaddeus (1975) who present results for $j \leq 5$ and $j^{\prime} \leq 5$. We found that at the densities and temperatures of interest, significant populations occurred of the levels up to $j=20$ so that an extrapolation of the cross section data was required.

For the rate coefficients $K_{j j^{\prime}}$ at temperature $T$ we adopted empirical representations of the form

$$
\begin{aligned}
K_{j j^{\prime}}= & a(\Delta j) \frac{g_{j^{\prime}}}{g_{j}}\left(1+\frac{\Delta E_{j j^{\prime}}}{k T}\right) \\
& \times \exp \left[-b(\Delta j)\left(\frac{\Delta E_{j j^{\prime}}}{k T}\right)^{1 / 2}\right],
\end{aligned}
$$

where $\Delta j=j-j^{\prime}, \Delta E_{j j^{\prime}}=2 h B\left[j(j+1)-j^{\prime}\left(j^{\prime}+1\right)\right]$ is the energy difference between levels $j$ and $j^{\prime}$ and $a$

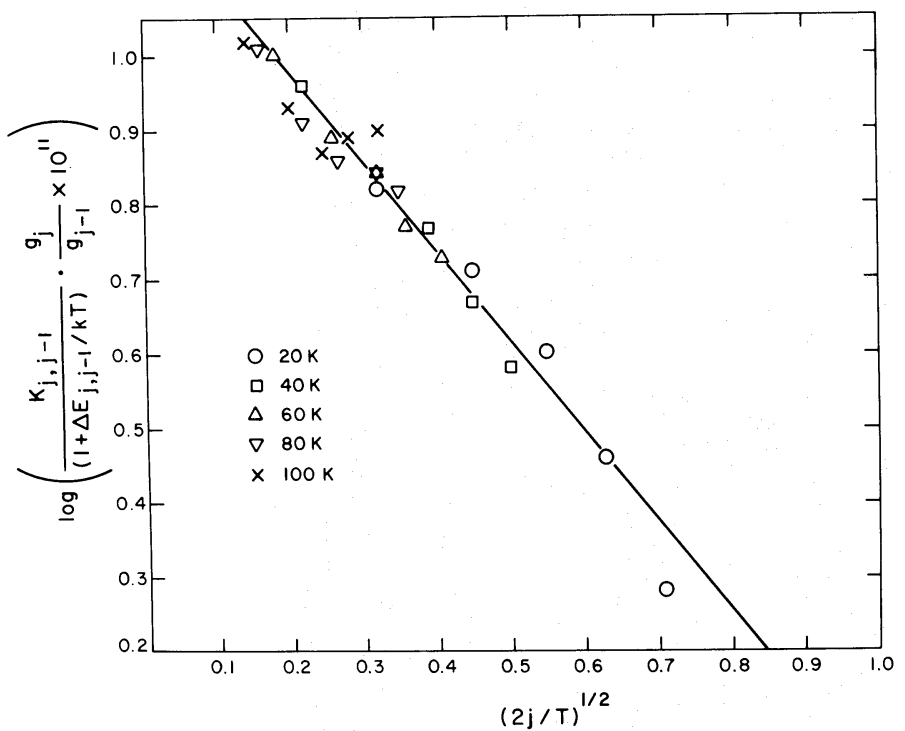

FIG. 1.-Fit of the rate coefficients $K_{j j^{\prime}}$ according to eq. (17) for $\Delta j=j-j^{\prime}=1$ 
and $b$ are functions only of $\Delta j$. Values of $a$ and $b$ for $\Delta j$ up to 5 are presented in Table 1 . The quality in of the representations is demonstrated in Figure 1 for $\therefore !$ the case $\Delta j=1$.

\section{NUMERICAL RESULTS}

Using a matrix-inversion technique, we have solved the equation of statistical equilibrium (1) for the level populations of ${ }^{12} \mathrm{CO}$ and ${ }^{13} \mathrm{CO}$ in spherical collapsing clouds. For ${ }^{12} \mathrm{CO}, B=57,700 \mathrm{MHz}$; for ${ }^{13} \mathrm{CO}$, $B=55,100 \mathrm{MHz}$. The dipole moment is 0.112 debyes (Burrs 1958). We adopted a fractional abundance $X\left({ }^{12} \mathrm{CO}\right)$ of $3 \times 10^{-5}$ for the main isotopic species and $X\left({ }^{13} \mathrm{CO}\right)$ of $3.4 \times 10^{-7}$ for the less abundant species; the adopted isotopic ratio is the terrestrial value $89: 1$. The parameter $\tau$ depends on the combination $X(\mathrm{CO}) n\left(\mathrm{H}_{2}\right) R / V$ so that an increase in $V / R$ is equivalent to a decrease in $X(\mathrm{CO})$. Thus both an increase in the collapse velocity and a decrease in the abundance of the molecules lower the opacity. The radiative transitions obey the selection rule that $j$ changes by unity and the coefficients $A_{j, j-1}$ and $B_{j, j-1}$ in (2) have been written down by Goldreich and Kwan (1974).

The rate of energy loss produced by emission in a transition from level $j$ to level $j-1$ is

$$
\begin{aligned}
\frac{d E_{j, j-1}}{d t}= & 2 h \mathrm{Bjn}_{j} A_{j, j-1} \beta_{j, j-1} \\
& \times\left[\frac{S_{j, j-1}-B\left(\nu_{j, j-1}, T_{\mathrm{BB}}\right)}{S_{j, j-1}}\right] .
\end{aligned}
$$

The factor in brackets reflects the fact that the rate of energy loss is measured with respect to the blackbody radiation field. In the absence of a background radiation field, the factor would be unity.

In a model A cloud the rate of energy loss is uniform throughout the cloud, but in model B clouds the rate is a function of the radial distance $r$. In discussing the results, we shall ordinarily compare the average energy loss rates, $\langle d E / d t\rangle$, defined according to

$$
\frac{\langle d E\rangle}{d t}=3 \int_{0}^{1} \rho^{2} \frac{d E(\rho)}{d t} d \rho,
$$

where $\rho=r / R$.

Table 2 shows the calculated level populations $n_{j}$ and energy loss rates $d E / d t$ for clouds with a molecular hydrogen density $n\left(\mathrm{H}_{2}\right)=2 \times 10^{4} \mathrm{~cm}^{-3}$, a kinetic temperature $T=25 \mathrm{~K}$, and a velocity-to-radius ratio $V / R=6 \mathrm{~km} \mathrm{~s}^{-1} \mathrm{pc}^{-1}$. Columns (1) and (2) compare the results for cloud model A computed with the collision rate coefficients, equation (17), and computed with the rate coefficients advocated by Goldreich and Kwan (1974). The populations of the lower $j$ levels approach thermal equilibrium and are essentially independent of the collision rates.

For thermalization to be achieved, radiative trapping must be significant and the optical depth $\tau$ exceeds unity (cf. § VI). Then in case A, we obtain, ignoring the background radiation field,

$$
\frac{d E_{j, j-1}}{d t}=4 \pi B\left(\nu_{j, j-1}, T\right) \frac{V}{R} \frac{\nu_{j, j-1}}{c},
$$

independent of the collision rate.

For the higher $j$ levels, which are not thermalized, $\tau<1$ and $\beta \simeq 1$, so that equation (18) becomes

$$
\frac{d E_{j, j-1}}{d t}=2 h B_{j n} A_{j, j-1} \text {. }
$$

The radiation field in the lines is then small, and to a useful approximation for the high $j$ levels

$$
n_{j} A_{j, j-1} \approx \sum_{j^{\prime}<j} n_{j^{\prime}} C_{j^{\prime}, j} .
$$

The corresponding rate of energy loss is given by

$$
\frac{d E_{j, j-1}}{d t} \approx 2 h B j \sum_{j^{\prime}<j} n_{j^{\prime}} C_{j^{\prime}, j},
$$

TABLE 2

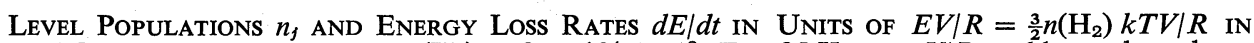
Molecular Clouds WITH $n\left(\mathrm{H}_{2}\right)=2 \times 10^{4} \mathrm{~cm}^{-3}, T=25 \mathrm{~K}$, AND $V \mid R=6 \mathrm{~km} \mathrm{~s}^{-1} \mathrm{pc}^{-1}$

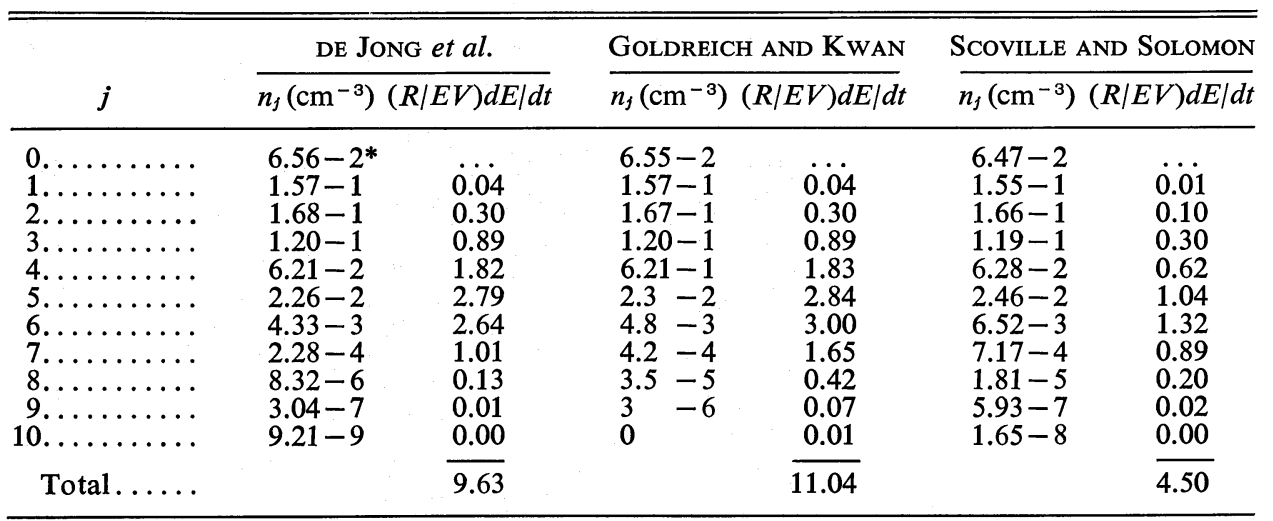

* Powers of 10. 
which is a function of the collision rates. Goldreich and Kwan (1974) adopted collisional cross sections that for the high $j$ levels are larger than those used by II us, and their computed energy loss rates are correspondingly larger than the values we have obtained. For molecules which are brought to thermal equilibrium less readily than is carbon monoxide, the choice of cross sections is still more critical.

The last column of Table 2 gives the energy losses in a plane-parallel cloud (Scoville and Solomon 1974) using our collisional rates. The substantial differences from the results for a spherical cloud in column (1) illustrate the effect of different geometries.

\section{CARBON MONOXIDE COOLING}

The calculated total rate of energy loss from the cloud in the carbon monoxide lines, $d E / d t=$ $\sum_{j} d E_{j, j-1} / d t$, is shown in Figure 2 as a function of the density $n\left(\mathrm{H}_{2}\right)$. Results are shown for cloud models $A$ and $B$ with kinetic temperatures of $20 \mathrm{~K}$ and $80 \mathrm{~K}$ and velocity-to-radius ratios of $10 \mathrm{~km} \mathrm{~s}^{-1} \mathrm{pc}^{-1}$ and $1 \mathrm{~km} \mathrm{~s}^{-1} \mathrm{pc}^{-1}$.

At low densities the cooling rates depend upon the density distribution but not on the velocity distribution, whereas at higher densities the cooling rates depend upon the magnitude of the velocity gradient but not on the density distribution, and models $\mathrm{A}$ and $B$ give similar results.

At low densities, the cloud becomes transparent and the levels are populated in thermal equilibrium at a temperature equal to the blackbody temperature $2.7 \mathrm{~K}$.

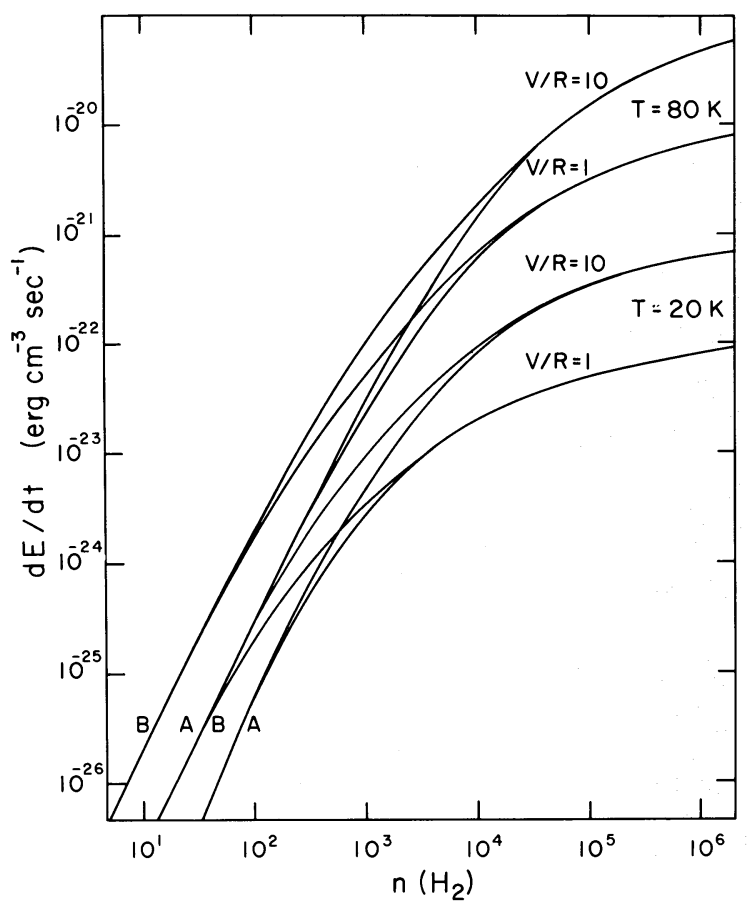

FIG. 2.-Total cooling rates $d E / d t$ as a function of the density, for cloud models $\mathrm{A}$ and $\mathrm{B}$ with kinetic temperatures of $20 \mathrm{~K}$ and $80 \mathrm{~K}$ and $V \mid R=1$ and $10 \mathrm{~km} \mathrm{~s}^{-1} \mathrm{pc}^{-1}$.
Collisional excitations from level $j$ to higher levels $j^{\prime}$ are followed immediately by downward radiative decays to level $j$, and the total cooling rate can be approximated by

$$
\frac{d E}{d t}=h B \sum_{j^{\prime}=0}^{\infty} \sum_{j^{\prime}>j} n_{j}\left(T_{\mathrm{BB}}\right) C_{j j^{\prime}}\left[j^{\prime}\left(j^{\prime}+1\right)-j(j+1)\right],
$$

where $n_{j}\left(T_{\mathrm{BB}}\right)$ is the thermal population of level $j$ at a temperature $T_{\mathrm{BB}}$. Thus the total cooling rate at low densities is proportional to $X(\mathrm{CO}) n^{2}\left(\mathrm{H}_{2}\right)$.

At high densities, the total cooling rate is given essentially by the sum of the energy loss rates in all the transitions that have been thermalized. Suppose $j_{m}$ is the highest level whose population is in thermal equilibrium. Then from equation (20),

$$
\frac{d E}{d t} \approx \sum_{j=1}^{j_{m}} 4 \pi B\left(\nu_{j, j-1}, T\right) \frac{V}{R} \frac{\nu_{j, j-1}}{c} .
$$

In most clouds, $2 h B j_{m}<k T$ and the Rayleigh-Jeans radiation law is appropriate so that equation (25) becomes

$$
\begin{aligned}
\frac{d E}{d t} & \approx \sum_{j=1}^{j_{m}} \frac{64 \pi}{c^{3}} B^{3} k T j^{3} \frac{V}{R} \\
& =\frac{16 \pi}{c^{3}} B^{3} k T \frac{V}{R} j_{m}^{2}\left(j_{m}+1\right)^{2}
\end{aligned}
$$

To derive $j_{m}$, we assume that the multilevel molecule can be described as a set of coupled two-level systems and we introduce the parameter

$$
\epsilon_{j, j-1}=\frac{C_{j, j-1}}{C_{j, j-1}+A_{j, j-1}\left[1-\exp \left(-h \nu_{j, j-1} / k T\right)\right]^{-1}}
$$

which defines the probability that a molecule in level $j$ is deactivated to level $j-1$ by collisions. The parameter $\epsilon_{j, j-1}$ is also the probability that a photon when it is scattered is removed from the radiation field and its energy is returned to the thermal energy of the gas.

If the optical depth $\tau_{j, j-1} \ll 1, \beta_{j, j-1}$ is approximately unity, photon trapping is unimportant, and level $j$ is populated by collisional excitation from level $j-1$ and depopulated by radiative emission to level $j-1$. If $\epsilon_{j, j-1}<\beta_{j, j-1}<1$, radiation trapping is important but the levels are not thermalized because the photons can escape before their energy is converted into thermal energy by collision processes. If $\beta_{j, j-1}<\epsilon_{j, j-1}$, the photons cannot escape, so that the levels are populated according to thermal equilibrium.

The value of $j_{m}$ is given by the thermalization condition $\beta_{j, j \rightarrow 1} \approx \epsilon_{j, j-1}$ or equivalently, $\tau_{j_{m}, j_{m}-1} \approx$ $\epsilon_{j_{m}, j_{m}-1}{ }^{-1}$. Since $C_{j_{m}, j_{m}-1}$ is very much smaller than 
$A_{j_{m}, j_{m}-1}$ in the physical conditions of interstellar clouds, the thermalization condition may be written

$$
\begin{aligned}
\frac{c^{3} A_{j_{m}, j_{m}-1}}{64 \pi B^{3} j_{m}{ }^{3}}\left(\frac{n_{j_{m}-1} g_{j_{m}}}{g_{j_{m}-1}}-n_{j_{m}}\right) \frac{R}{V} \\
=\frac{A_{j_{m}, j_{m}-1}}{C_{j_{m}, j_{m}-1}\left[1-\exp \left(-2 h B j_{m} / k T\right)\right]} .
\end{aligned}
$$

Assuming that $2 h B j_{m}<k T$ and that $n_{j_{m}}$ and $n_{j_{m}-1}$ have values according to thermal equilibrium, we obtain the expression

$$
\begin{aligned}
& j_{m}\left(j_{m}+1\right)=\frac{k T}{h B} \\
& \times \ln \left[\frac{c^{3} h^{2}}{16 \pi k^{2}} \frac{R}{V} \frac{\left(2 j_{m}+1\right) X(\mathrm{CO}) n^{2}\left(\mathrm{H}_{2}\right) K_{j_{m}, j_{m}-1}}{B j_{m}{ }^{2} T^{2}}\right]
\end{aligned}
$$

from which $j_{m}$ may be determined. The result is plausible because we expect that in thermal equilibrium the levels will be populated up to a $j$ value of about $(k T / h B)^{1 / 2}$. The effect of radiation trapping is contained in the logarithmic factor.

For the cooling rate, we obtain

$$
\begin{aligned}
\frac{d E}{d t}= & \frac{16 \pi k^{3}}{h^{2} c^{3}} B T^{3} \frac{V}{R} \\
& \times \ln ^{2}\left[\frac{c^{3} h^{3}}{16 \pi k^{2}} \frac{R}{V} X(\mathrm{CO}) \frac{n^{2}\left(\mathrm{H}_{2}\right)\left(2 j_{m}+1\right) K_{j_{m}, j_{m}-1}}{B T^{2} j_{m}{ }^{2}}\right],
\end{aligned}
$$

which implies that at higher densities $d E / d t$ is proportional to $T^{3} V / R$. The numerical data are consistent with the low and high density limits that we have derived.

The formula (30) demonstrates also that the cooling rate increases slowly as the density increases and that at high densities cooling from transitions in molecules with large level separations is relatively enhanced (Dalgarno et al. 1974).

In clouds without velocity gradients that have the same radius $R$ and a turbulent velocity of the order of $V$, the total cooling is still of the same order as equation (30) because the escape probability at large optical depths is approximately $\tau^{-1}\left[\ln \left(\tau / \pi^{1 / 2}\right)\right]^{-1 / 2}$ which differs only by a logarithmic factor from the escape probability in a collapsing cloud.

The limiting formulae provide a simple interpretation of the differences between models A and B. In model B, the local cooling rate is a function of position within the cloud. At low densities, the cooling rate increases with $n\left(\mathrm{H}_{2}\right)^{2}$ so that the average cooling rate in model B exceeds that in model A. At high densities, the cooling depends on density only through the logarithmic factor in equation (30) and the differences between models A and B are small.

\section{LINE PROFILES AND BRIGHTNESS TEMPERATURES}

The cloud models are distinguished by different line profiles. Figure 3 indicates the loci of volume elements

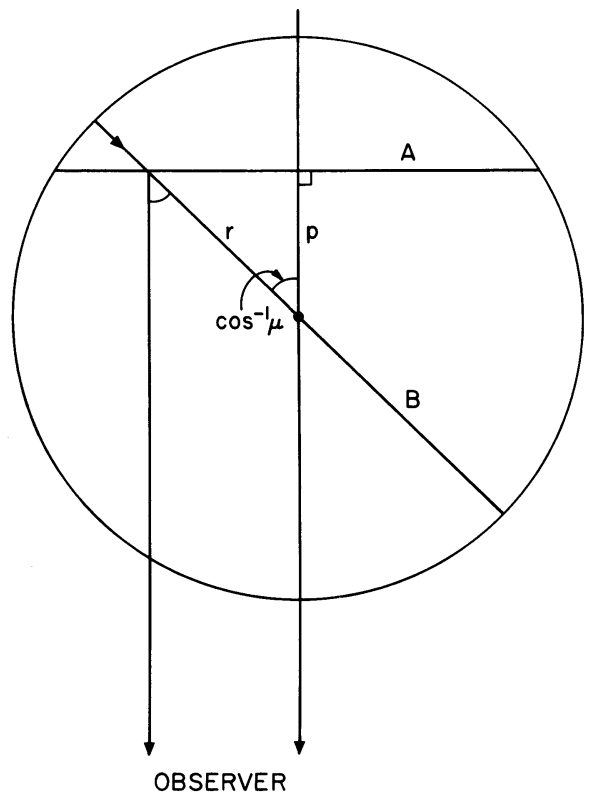

FIG. 3.-Loci of volume elements within spherical clouds with equal radial velocities $v_{0}$ toward the observer. In cloud $\mathrm{A}$, $v_{0}=V p / R ;$ in cloud $\mathrm{B}, v_{0}=V p / r$.

within the cloud which have equal velocities $v_{0}$ in the direction of an observer. In model A, $v_{0}=v(r) p / r=$ $V p / R$ and the photons reaching the observer with frequencies corresponding to velocities $\pm v_{0}$ are emitted in planes perpendicular to the line of sight at distances $\pm p$ from the plane through the cloud center. In model $\mathrm{B}, v_{0}=V p / r$ and the photons are emitted in planes making angles $\pm \cos ^{-1}(p / r)$ with the plane through the center if they are to have frequencies corresponding to a velocity $\pm v_{0}$ toward the observer.

We assume now that the cloud fills the beam of the telescope, and for simplicity we neglect the background radiation field. Then the flux emitted by a volume element at radial distance $r$ in the direction $\mu$ is $\pi S(r)\{1-\exp [-\tau(r, \mu)]\}$. Integrating over the planes of equal $v_{0}$, we obtain for the average flux, $F\left(v_{0}\right)$, across the surface of cloud model A,

$$
\begin{aligned}
F\left(v_{0}\right) & =\frac{1}{\pi R^{2}} \int_{0}^{R\left|1-\mu^{2}\right|^{1 / 2}} d t 2 \pi t \pi S[1-\exp (-\tau)] \\
& =\pi S[1-\exp (-\tau)]\left(1-v_{0}^{2} / V^{2}\right)
\end{aligned}
$$

which may be rewritten in the form

$$
F\left(v_{0}\right)=\frac{1}{4} \frac{d E}{d t} \frac{R}{V} \frac{c}{\nu_{0}}\left(1-\frac{v_{0}^{2}}{V^{2}}\right) .
$$

The blackbody radiation field can be correctly included in equation (31) by substituting $d E / d t$ according to equation (18). The brightness temperature $T_{b}\left(v_{0}\right)$ may be defined by the ratio

$$
T_{b}\left(v_{0}\right)=F\left(v_{0}\right) / B(\nu, T), \quad \nu=\nu_{0}\left(1 \pm v_{0} / c\right),
$$




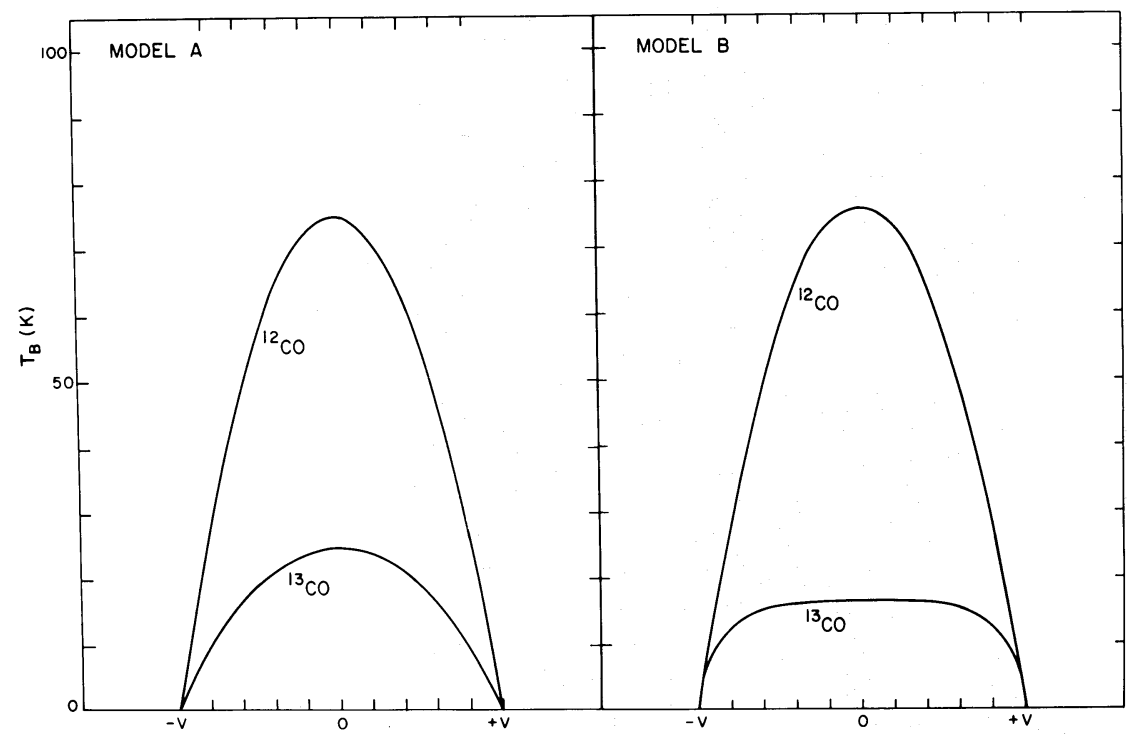

FIG. 4.-Line profiles of the $j=1 \rightarrow 0$ lines of ${ }^{12} \mathrm{CO}$ and ${ }^{13} \mathrm{CO}$ molecules for cloud model A (Fig. $4 a$ ) and for cloud model B (Fig. $4 b$ ). The cloud parameters are $T=80 \mathrm{~K}, n\left(\mathrm{H}_{2}\right)=10^{5} \mathrm{~cm}^{-3}, X\left({ }^{12} \mathrm{CO}\right)=3 \times 10^{-5}, X\left({ }^{13} \mathrm{CO}\right) / X\left({ }^{12} \mathrm{CO}\right)=1 / 89$, and $V / R=$ $1 \mathrm{~km} \mathrm{~s}^{-1} \mathrm{pc}^{-1}$.

where $B(\nu, T)$ is the Rayleigh-Jeans radiation field, so that

$$
T_{b}\left(v_{0}\right)=\frac{c^{3}}{8 \pi k v_{0}^{3}} \frac{d E}{d t} \frac{R}{V}\left(1-\frac{v_{0}^{2}}{v^{2}}\right) .
$$

By a similar analysis we obtain for cloud model B

$$
T_{b}\left(v_{0}\right)=\frac{c^{3}}{8 \pi k \nu^{3}} \frac{R}{V} \frac{\left\langle d E\left(v_{0}\right)\right\rangle}{d t},
$$

where $\left\langle d E\left(v_{0}\right) / d t\right\rangle$ is the mean energy loss rate averaged over planes with equal $v_{0}$,

$$
\frac{\left\langle d E\left(v_{0}\right)\right\rangle}{d t}=\frac{1}{\pi R^{2}} \int_{0}^{R} 2 \pi r \frac{d E\left(r, v_{0}\right)}{d t} d r,
$$

and $d E\left(r, v_{0}\right) / d t$ is given by equation (18) with $\beta\left(r, v_{0}\right)=$ $\left\{1-\exp \left[-\tau\left(r, v_{0}\right)\right]\right\} / \tau\left(r, v_{0}\right)$ and

$$
\tau\left(r, v_{0}\right)=\frac{A_{i j} c^{3} n_{i}}{8 \pi \nu^{3}}\left(\frac{n_{j} g_{i}}{n_{i} g_{j}}-1\right) \frac{r}{V} \frac{1}{\left(1-v_{0}^{2} / V^{2}\right)}
$$

Profiles of the $j=1-0$ lines of ${ }^{12} \mathrm{CO}$ and ${ }^{13} \mathrm{CO}$ molecules are illustrated in Figure 4 for cloud $\mathrm{A}$ and cloud B models with kinetic temperature $80 \mathrm{~K}$, mean density $n\left(\mathrm{H}_{2}\right)=10^{5} \mathrm{~cm}^{-3}$, a fractional abundance of ${ }^{12} \mathrm{CO}$ of $3 \times 10^{-5}$, an isotopic ratio for ${ }^{12} \mathrm{CO}$ to ${ }^{13} \mathrm{CO}$ of 89 , and a velocity-to-radius ratio of $1 \mathrm{~km} \mathrm{~s}^{-1} \mathrm{pc}^{-1}$. In neither case does the brightness temperature reach $80 \mathrm{~K}$ essentially because the Rayleigh-Jeans approximation is not strictly applicable and because the zerointensity level for the lines is determined relative to the $2.7 \mathrm{~K}$ blackbody radiation field.

Figure 4 shows that the profiles of the ${ }^{12} \mathrm{CO}$ lines are similar in the two cloud models. This similarity stems from the adopted high density which results in large optical depths and according to equations (33)(36) makes the brightness temperatures independent of the cloud model. The weaker ${ }^{13} \mathrm{CO}$ lines have dissimilar profiles because the fluxes at the small values of $\tau$ that are appropriate to the ${ }^{13} \mathrm{CO}$ lines are proportional to the number of molecules contributing to $T_{b}\left(v_{0}\right)$ and that number varies as $\left(1-v_{0}^{2} / V^{2}\right)$ in model A but is constant in model B. (See also the loci of $v_{0}$ in Fig. 3.) We note that high gas densities are required to reproduce the brightness temperature ratios of about 3 observed in many sources.

The observed line profiles of the main and the isotopic lines are often similar in shape, though the widths of the isotope lines are about half those of the principal line. Neither of our models is consistent with the observations. The introduction of a gradient in the density in model A would make the isotopic line narrower than the main line since the isotopic line would be formed in a region more concentrated toward the cloud center.

The telescope beam is often smaller than the cloud size. The measured ${ }^{12} \mathrm{CO}$ line profiles are no longer similar for cloud models $\mathrm{A}$ and $\mathrm{B}$, although both become more flat-topped.

In Figure 5, we show the peak brightness temperatures of the $1-0{ }^{12} \mathrm{CO}$ line as functions of density at two values of the ratio $V / R=1$ and $10 \mathrm{~km} \mathrm{~s}^{-1} \mathrm{pc}^{-1}$ for cloud models $A$ and $B$ with $T=80 \mathrm{~K}$ and $X\left({ }^{12} \mathrm{CO}\right)=3 \times 10^{-5}$. Although at the intermediate densities the 1-0 transition is thermalized in the core of cloud B while it is not yet thermalized in cloud A, the averaging procedure makes the peak brightness temperature at those densities higher for cloud $\mathrm{A}$ than for cloud $\mathbf{B}$.

In Figure 6, we show the peak brightness temperatures of the $1-0^{12} \mathrm{CO}$ line for cloud $\mathrm{B}$ at $T=20 \mathrm{~K}$ and $80 \mathrm{~K}$ and $V / R=1$ and $10 \mathrm{~km} \mathrm{~s}^{-1} \mathrm{pc}^{-1}$ as functions 


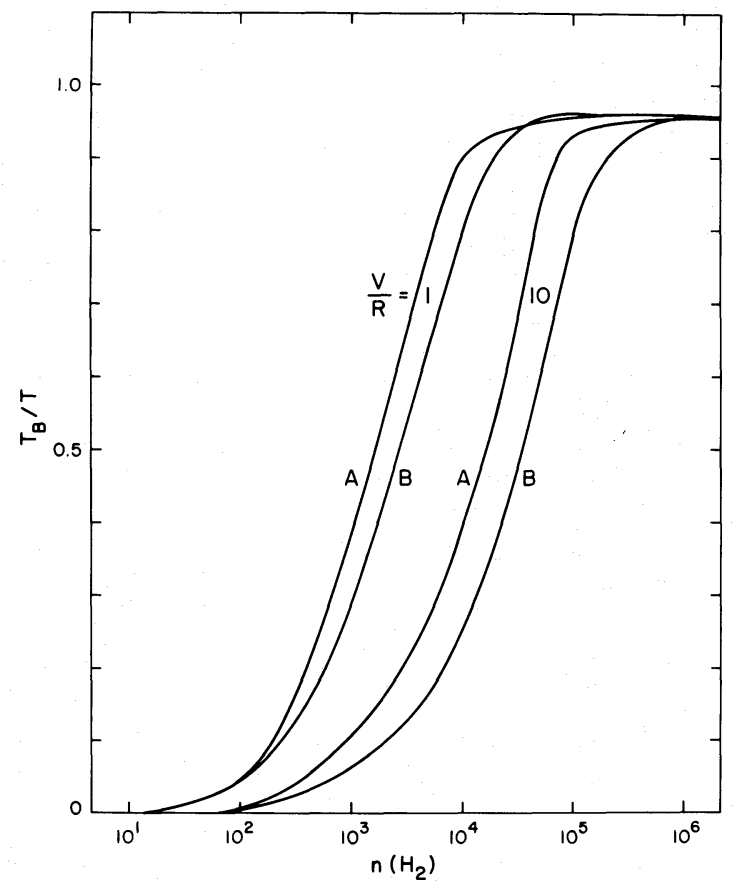

FIG. 5.-Peak brightness temperature of the $j=1 \rightarrow 0^{12} \mathrm{CO}$ for cloud models $\mathrm{A}$ and $\mathrm{B}$ as functions of the density for $V \mid R=$ 1 and $10 \mathrm{~km} \mathrm{~s}^{-1} \mathrm{pc}^{-1}, T=80 \mathrm{~K}$, and $X\left({ }^{12} \mathrm{CO}\right)=3 \times 10^{-5}$.

of density. Figures 5 and 6 may be useful in the interpretation of observations of ${ }^{12} \mathrm{CO}$ lines, bearing in mind that the results scale with $X(\mathrm{CO}) R / V$.

\section{BRIGHTNESS TEMPERATURE RATIOS}

Figure 7 illustrates the calculated ratios of the brightness temperatures of the $2-1$ and $1-0$ lines of

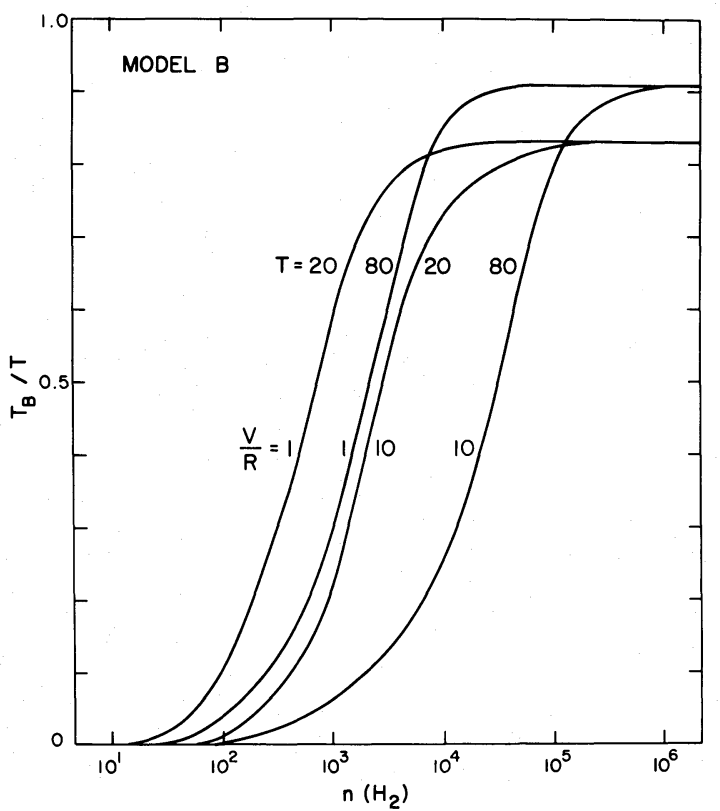

FIG. 6.-Peak brightness temperatures of the $j=1 \rightarrow 0{ }^{12} \mathrm{CO}$ line for cloud model $\mathrm{B}$ as functions of the density for $T=20 \mathrm{~K}$ and $80 \mathrm{~K}$ and $V \mid R=1$ and $10 \mathrm{~km} \mathrm{~s}^{-1} \mathrm{pc}^{-1}$.

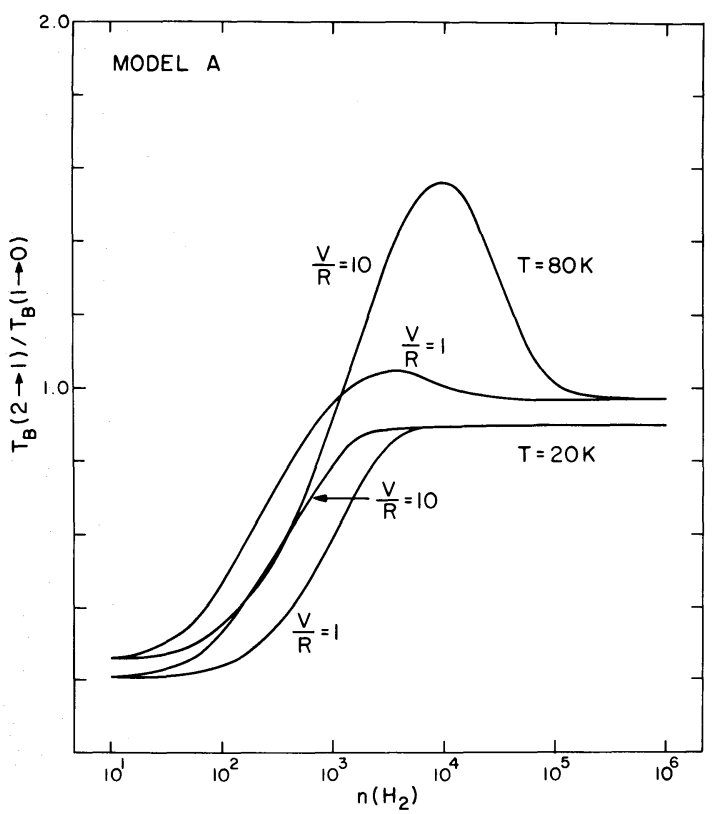

Fig. 7.-Calculated ratios of the brightness temperatures of the $j=2 \rightarrow 1$ and $j=1 \rightarrow 0$ lines of ${ }^{12} \mathrm{CO}$ for cloud model $\mathrm{A}$ as a function of density for $V \mid R=1$ and $10 \mathrm{~km} \mathrm{~s}^{-1} \mathrm{pc}^{-1}$ and $T=20 \mathrm{~K}$ and $80 \mathrm{~K}$.

${ }^{12} \mathrm{CO}$ for $V / R=1$ and $10 \mathrm{~km} \mathrm{~s}^{-1} \mathrm{pc}^{-1}$ and for $T=$ $20 \mathrm{~K}$ and $80 \mathrm{~K}$ as a function of particle density in cloud model A.

Of particular interest is the case with $V / R=10 \mathrm{~km}$ $\mathrm{s}^{-1} \mathrm{pc}^{-1}$ and $T=80 \mathrm{~K}$, for which the ratio exceeds unity for densities between $10^{3} \mathrm{~cm}^{-3}$ and $10^{5} \mathrm{~cm}^{-3}$. Calculations show that for this range of densities, $\tau_{21}>\epsilon_{21}{ }^{-1}$ while $\tau_{10} \leqslant \epsilon_{10}{ }^{-1}$. Thus because of the enhanced $j=2 \rightarrow 0$ collision rate coefficient the $2-1$ transition thermalizes at a lower density than does the 1-0 transition.

This effect can be exploited to construct a model of the Orion molecular cloud observed in the ${ }^{12} \mathrm{CO}$ lines by Phillips, Jefferts, and Wannier (1973). They presented the peak antenna temperatures of the $2-1$ and 1-0 lines along a strip in right ascension through the center of the Orion molecular cloud. The results are reproduced in Figure 8.

In model $\mathrm{A}$, the peak brightness temperatures are emitted in the center plane of the cloud. We suggest that the results of Figure 8 can be reproduced by a cloud with a density gradient that reaches a maximum at the cloud center. At the center the density is high and the ratio of the 2-1 and 1-0 brightness temperatures is unity. With increasing distance from the center the density falls and the brightness temperature ratio increases. The ratio should then pass through a maximum and return to a value of unity before falling off to smaller values.

The observations (Phillips et al. 1974) also show that the 2-1 brightness temperature passes through maxima at intervals in right ascension of about 11' on both sides of the center. If the increases are due to clumpiness (Phillips et al. 1974), the clumps have 


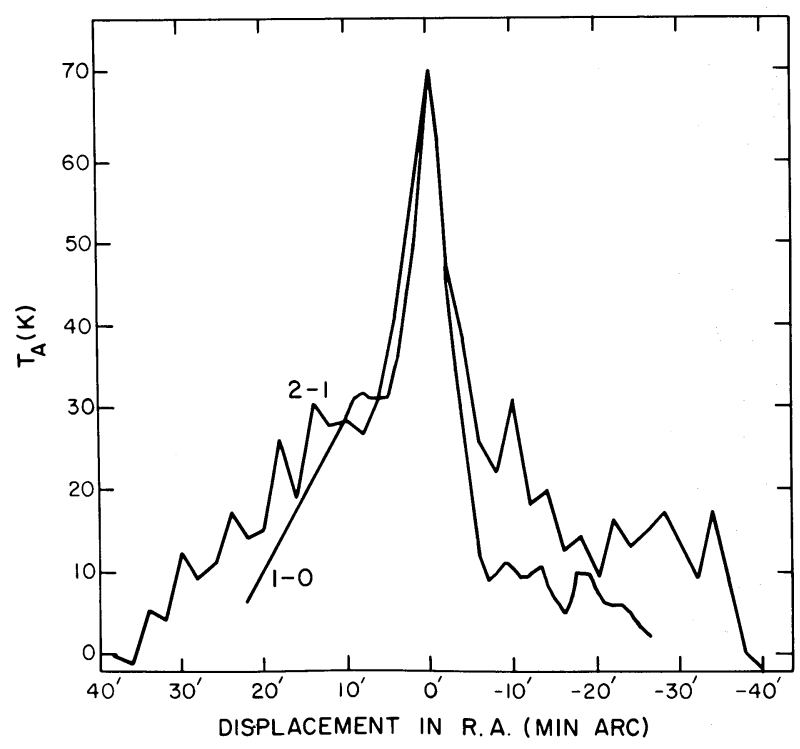

FIG. 8.-Peak antenna temperatures of the $j=2 \rightarrow 1$ and $j=1 \rightarrow 0$ line along a strip in right ascension through the center of the Orion molecular cloud (reproduced from Phillips et al. 1973).

densities of the order of $10^{4} \mathrm{~cm}^{-3}$, between the clumps the densities are of the order of $10^{3} \mathrm{~cm}^{-3}$, and at the cloud center the density is about $10^{5} \mathrm{~cm}^{-3}$. However, a variable velocity gradient provides a possible alternative interpretation (Linke and Wannier 1974).

A recent model of the Orion molecular cloud by Gerola and Sofia (1975) which in fact includes both a velocity gradient and a density gradient in the manner that we suggest, fails to reproduce the observed ratio of the $2 \rightarrow 1$ and $1 \rightarrow 0$ brightness temperatures of the $\mathrm{CO}$ lines. The failure may stem from their adopted collision rates in which the $j=2 \rightarrow 0$ rate is not enhanced.

\section{INFRARED-EMISSION FROM CARBON MONOXIDE}

Most of the energy emitted by carbon monoxide appears in the far-infrared region of the spectrum and may become detectable. We have calculated the line intensities for a typical dense interstellar molecular cloud, and we have compared them with the intensity of the dust continuum radiation that may occur. We adopted cloud model A with $n\left(\mathrm{H}_{2}\right)=10^{4} \mathrm{~cm}^{-3}$, $R=3 \mathrm{pc}, V=3 \mathrm{~km} \mathrm{~s}^{-1}$, and $X\left({ }^{13} \mathrm{CO}\right)=3 \times 10^{-5}$ at a distance of $3 \mathrm{kpc}$.

The flux received at the Earth averaged over a particular line with frequency $\nu$ is

$$
\begin{aligned}
F_{v L} & =\frac{R^{3}}{3 D^{2}} \frac{d E}{d t} \frac{c}{V \nu} \\
& =2.9 \times 10^{24} \lambda \frac{d E}{d t} v^{\prime 2} \mathrm{Jy}
\end{aligned}
$$

when $\lambda$ is the wavelength in microns and $v^{\prime}$ is the angular radius of the cloud in arc minutes. The computed values of $F_{v L}$ are given in Table 3 for ${ }^{12} \mathrm{CO}$ and Table 4 for ${ }^{13} \mathrm{CO}$, for clouds at temperatures of $20 \mathrm{~K}$ and $80 \mathrm{~K}$.

The flux of continuum radiation emitted by the dust may be written

$$
\begin{aligned}
F_{v C} & =\frac{\pi R^{2}}{4 \pi D^{2}} \pi B(\nu, T)\left[1-\exp \left(-t_{v}\right)\right] \\
& =6.6 \times 10^{-8} v^{\prime 2} B(\nu, T)\left\{1-\exp \left(-t_{v}\right)\right\}
\end{aligned}
$$

where

$$
t_{v}=\frac{4}{3} R n_{d} \pi a^{3} \kappa_{m}\left(\nu / \nu_{m}\right)^{2}
$$

is the optical depth of particles small compared with the wavelength, $n_{d}$ is the dust particle density, $a$ is the radius, and $\kappa_{m}\left(\nu / \nu_{m}\right)^{2}$ is the absorption coefficient of the

TABLE 3

Predicted Infrared ${ }^{12} \mathrm{CO}$ Line Intensities and Dust ContinuUm Intensities from a Cloud WITH $n_{\mathrm{H}_{2}}=10^{4} \mathrm{~cm}^{-3}, R=3 \mathrm{pc}$, AND $V / R=1 \mathrm{~km} \mathrm{sec}^{-1} \mathrm{pc}^{-1}$ AT DISTANCE $D=3 \mathrm{kpc}$

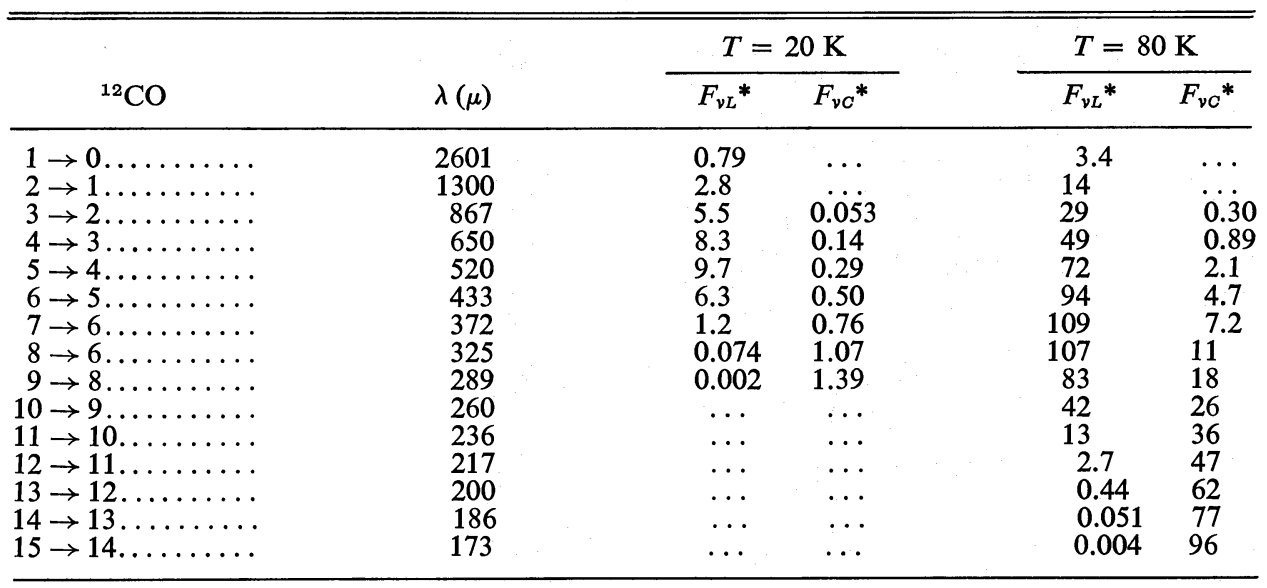

$* 1000 \mathrm{Jy} \operatorname{arcmin}^{-2}$. 
DE JONG, CHU, AND DALGARNO

TABLE 4

Predicted Infrared ${ }^{13} \mathrm{CO}$ Line Intensities for the Cloud Model of Table 3

\begin{tabular}{|c|c|c|c|c|c|}
\hline \multirow[b]{2}{*}{${ }^{13} \mathrm{CO}$} & \multirow[b]{2}{*}{$\lambda(\mu)$} & \multicolumn{2}{|c|}{$T=20 \mathrm{~K}$} & \multicolumn{2}{|c|}{$T=80 \mathrm{~K}$} \\
\hline & & $F_{v L} *$ & $F_{v C^{*}}$ & $F_{v L}{ }^{*}$ & $F_{v C^{*}}$ \\
\hline 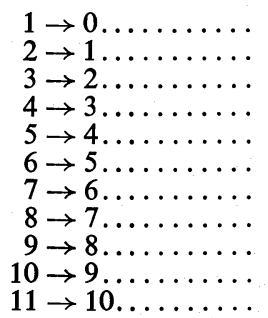 & $\begin{array}{r}2722 \\
1361 \\
907 \\
681 \\
544 \\
454 \\
389 \\
340 \\
302 \\
272 \\
247\end{array}$ & $\begin{array}{l}0.33 \\
1.7 \\
2.5 \\
1.6 \\
0.39 \\
0.047 \\
0.003 \\
\ldots \\
\cdots \\
\cdots \\
\ldots\end{array}$ & $\begin{array}{l}\cdots \\
0.045 \\
0.12 \\
0.25 \\
0.43 \\
0.67 \\
\cdots \\
\cdots \\
\cdots\end{array}$ & $\begin{array}{l}0.24 \\
2.7 \\
7.1 \\
10.1 \\
9.2 \\
5.5 \\
2.4 \\
0.75 \\
0.19 \\
0.038 \\
0.006\end{array}$ & $\begin{array}{l}\cdots \\
\cdots \\
0.80 \\
1.76 \\
3.5 \\
6.1 \\
10 \\
15 \\
22 \\
31\end{array}$ \\
\hline
\end{tabular}

$* 1000 \mathrm{Jy} \operatorname{arcmin}^{-2}$.

dust material. We adopted the parameters $n_{d}=$ $2.4 \times 10^{-12} n\left(\mathrm{H}_{2}\right), a=1.5 \times 10^{-5} \mathrm{~cm}, \kappa_{m}=10^{4} \mathrm{~cm}^{-1}$, and $\lambda_{m}=20 \mu$, so that $t_{v}=32\left(\nu / \nu_{m}\right)^{2}$.

Values of $F_{v C}$ are given in Tables 3 and 4 for the case that the dust and gas have equal temperatures (cf. Goldreich and Kwan 1974). The infrared emission at shorter wavelengths is dominated by dust radiation and high resolution will be necessary to detect the lines. At larger wavelengths, the lines are the main source of the emission.

We acknowledge useful discussions with Dr. George Rybicki. The collision rate data were kindly provided to us by Dr. Sheldon Green prior to publication. This research was partly supported by the National Science Foundation.

\section{REFERENCES}

Burrus, C. A. 1958, J. Chem. Phys., 28, 427.

Castor, J. I. 1970, M.N.R.A.S., 149, 111.

Compaan, A., Langer, W. D., Eden, D., and Swinney, H. L. 1973, Ap. J. (Letters), 185, L105.

Dalgarno, A., de Jong, T., Oppenheimer, M., and Black, J. H. 1974, Ap. J. (Letters), 192 , L37.

Gerola, H., and Sofia, S. 1975, Ap. J., 196, 473.

Glassgold, A. E., and Langer, W. D. 1973, Ap. J. (Letters), 179, L147.

Goldreich, P., and Kwan, J. 1974, Ap. J., 189, 441.

Goldsmith, P. F. 1972, Ap. J., 176, 597.

Green, S., and Thaddeus, P., 1975, to be published.

Larson, R. B. 1969, M.N.R.A.S., 145, 271.

Linke, R. A., and Wannier, P. G. 1974, Ap. J. (Letters), 193, L41.
Lucy, L. B. 1971, Ap. J., 163, 95.

Phillips, T. G., Jefferts, K. B., and Wannier, P. G. 1973, Ap. J. (Letters), 186, L19.

Phillips, T. G., Jefferts, K. B., Wannier, P. G., and Ade, P. A. R. 1974, Ap. J. (Letters), 191, L31.

Rybicki, G. B. 1970, in Spectrum Formation in Stars with Steady-State Extended Atmospheres, NBS Spec. Pub., 332, 87.

Scoville, N. Z., and Solomon, P. M. 1974, Ap. J. (Letters), 187, L67.

Sobolev, V. V. 1960, Moving Envelopes of Stars (Cambridge: Harvard University Press).

SHIH-I CHU: JILA, University of Colorado, Boulder, CO 80302

A. DALGaRno: Center for Astrophysics, Harvard College Observatory and Smithsonian Astrophysical Observatory, 60 Garden Street, Cambridge, MA 02138

T. DE JONG: Sterrewacht, Leiden, The Netherlands 